\title{
Cerebral aspergilloma
}

\author{
BetTy M. Partridge \\ M.A., Ph.D. \\ A. T. L. CHIN \\ M.A., M.B., B.Ch. \\ Microbiology Department, Charing Cross Hospital Medical School, London W6 8RF
}

\begin{abstract}
Summary
This is the report on a fatal case of aspergilloma with intracranial mycotic aneurysm associated with Aspergillus flavus infection, confirmed by culture and serology. Drug therapy is discussed.

\section{Introduction}

Over the last decade extra-pulmonary aspergillosis has been reported with increasing frequency from the Middle and Far East. In the late 60s, 17 cases of paranasal aspergilloma, some with orbital involvement, were reported from the Sudan; Aspergillus flavus was found to be the causative organism in all 17 (Miloshev et al., 1966, 1969). A further 60 cases have been described from North Sudan where A. flavus is a common organism in the soil (Mahgoub,
\end{abstract} 1977).

Only 11 cases of solitary brain aspergilloma had been reported by $1977 ; 3$ of these, including one due to $A$. flavus, occurred in Thailand (Visudhiphan, Bunyaratavej and Khantanaphar, 1973), 2 in India (Venugopal et al., 1977).

Other aspergilli implicated in central nervous system infection have been $A$. fumigatus, $A$. oryzae, A. amstelodami, A. sydowi, A. glaucus, A. niger, A. candidus and $A$. versicolor.

The present case describes an $A$. flavus infection of the brain in a young man from Saudi Arabia.

\section{Case report}

An 18-year-old carpenter from Saudi Arabia, presented with blindness and progressive pain and swelling of the left eye. His symptoms had started more than 2 years previously and visual loss was complete within 6 months.

On examination he had a left ptosis and a left axial proptosis with limitation of external ocular movements of the left eye in all directions. The left pupil was dilated and fixed and there was left optic disc pallor. There was diminution to touch and pinprick over the first and second divisions of the left trigeminal nerve. The central nervous system was otherwise normal.

In view of the history and presentation, a retro- orbital tumour with an intracranial extension was suspected. A mild left frontal abnormality was noted on the EEG. The skull X-ray showed an enlarged left optic foramen with ill-defined left superior orbital fissure. The CAT scans demonstrated the presence of a very extensive abnormality on the left side which extended from the posterior ethmoid air cells forwards and laterally into the left orbit and posteriorly into the sphenoid sinus, through the sphenoid bone into the middle cranial fossa. The lesion had a similar density to the brain. Also noted was oedema of the adjacent temporal lobe, some compression of the brain stem at the level of the cerebral peduncle and slight mid-line shift.

A left fronto-temporal craniotomy was performed. A mass of firm tissue adherent to the frontal lobe, extending forwards but not in contact with the left optic nerve and bulging posteriorly into the middle cranial fossa, was excised.

\section{Laboratory investigations \\ Histopathology}

A preliminary report on frozen sections of the intercranial mass indicated a granuloma, possibly a tuberculoma. Stained sections confirmed a granulomatous reaction including numerous multinucleate giant cells. Central areas of necrosis were seen and other areas revealed a prominent infiltration of plasma cells and eosinophils. Stains for fungi showed the presence of numerous branching, septate, hyaline filaments suggestive of Aspergillus sp. (Fig. 1). Many of the fungal fragments were within giant cells. Stains for acid-fast bacilli were negative.

\section{Mycology}

An emulsion of the granulomatous tissue was plated out on peptone-dextrose, blood, MacConkey lactose bile salt and chocolate agars incubated at $37^{\circ} \mathrm{C}$. Heavy growths of a grey-green mould grew on all plates within $24 \mathrm{hr}$ and was identified as Aspergillus sp. No other organisms were grown. Subcultures of the fungus on to peptone-dextrose agar plates subsequently developed the rich green 


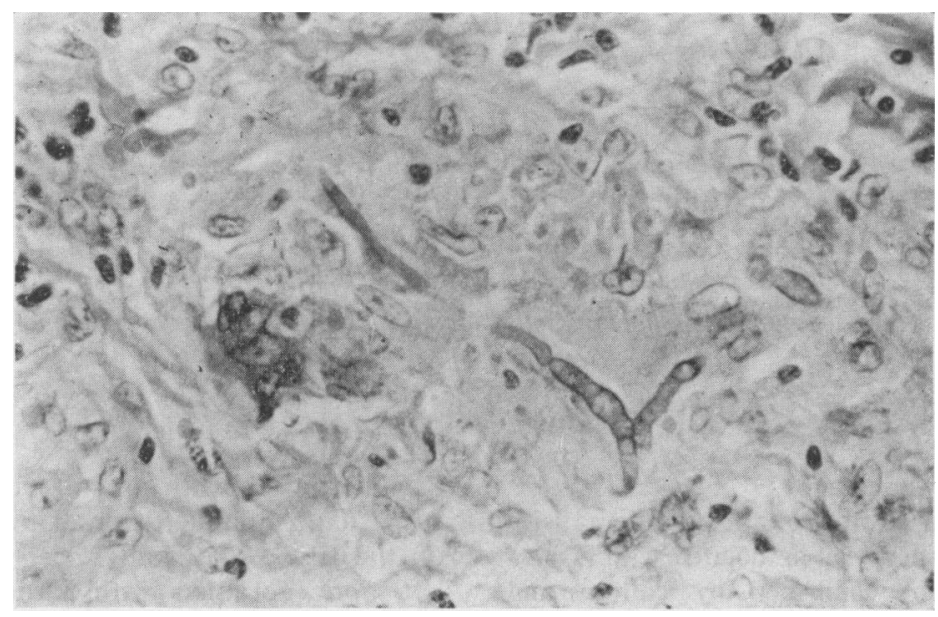

FIG. 1. Intracranial mass (periodic-acid-Schiff, $\times 350$ ).

floccose growth of $A$. flavus. Microscopically, the conidal heads were radiate to slightly columnar with rough-walled conidia; stalks were rough. Identification was confirmed by other laboratories.

\section{Serology}

Serum was screened for precipitins to Aspergillus antigens and found to be positive to A. flavus. An extract prepared from the patient's own isolate gave the strongest precipitin reaction, thus confirming diagnosis. Precipitin tests against $A$. fumigatus, $A$. niger and $A$. terreus were negative, although a positive precipitin was recorded against $A$. nidulans, indicating some cross reaction.

\section{Sensitivity tests}

Comparative tests were made using the method of incorporating drugs into agar plates, light spore inocula and incubation at $37^{\circ} \mathrm{C}$. Results showed that the patient's isolate of $A$. flavus was insensitive to $10 \mu \mathrm{g} / \mathrm{ml}$ of amphotericin B. Of the imidazoles, miconazole inhibited the isolate at $1 \mu \mathrm{g} / \mathrm{ml}$ and econazole at a level of $0 \cdot 1 \mu \mathrm{g} / \mathrm{ml}$.

\section{Toxin production}

No aflatoxins could be detected in cultures of the patient's isolate.

\section{Management}

Postoperatively, pending laboratory investigations, amphotericin B was given both intraventricularly and intravenously. For intraventricular administration the drug was diluted with CSF, an initial dose of $0.025 \mathrm{mg}$ being given with a daily increment of $0.025 \mathrm{mg}$. For intravenous infusion $12.5 \mathrm{mg}$ of the drug in $5 \%$ dextrose was given over a period of $6 \mathrm{hr}$, increasing the dose by $2.5 \mathrm{mg}$ daily. The side-effects following intraventricular amphotericin B were most marked, necessitating the use of antihistamines and antipyretics. Treatment was maintained for -8 days.

One week after the initial therapy, the patent complained of pain around the back of the heado

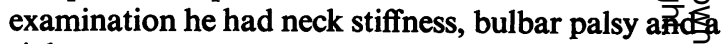
right upper motor neurone lesion. The CAT scan showed ventricular dilatation with intraventriculor haemorrhage in lateral, third and fourth ventricles. The patient's condition deteriorated and he died $\overrightarrow{\text { gf }}$ the fifteenth postoperative day.

\section{Post-mortem examination}

Post-mortem revealed a swollen and oedematous brain with a soft left temporal lobe. Dissection of the formalin-fixed brain and microscopy revealed ${ }_{3}$ ruptured mycotic aneurysm of the rostral end of a vertebral artery. The leptomeninges in this region were filled with chronic inflammatory and granulematous exudate, occasional hyphae of a fungis, morphologically compatible with Aspergillus, and recent haemorrhage. The basilar artery showed $\cong$ fairly cellular intimal fibrosis, focal destruction of the internal elastica with many attendant multinucleated giant cells and many fungal hyphae in the media (Fig. 2). In one vertebral artery the lesion was of increased severity with necrosis of the medien, rupturing and splaying out of part of the wall of the artery, with haemorrhage into the surrounding tissues. No other abnormality was seen.

\section{Discussion}

Aspergilli are widely distributed in nature and $\stackrel{\mathbb{R}}{a}$ among the commonest fungi in soil. In the Sudå, 


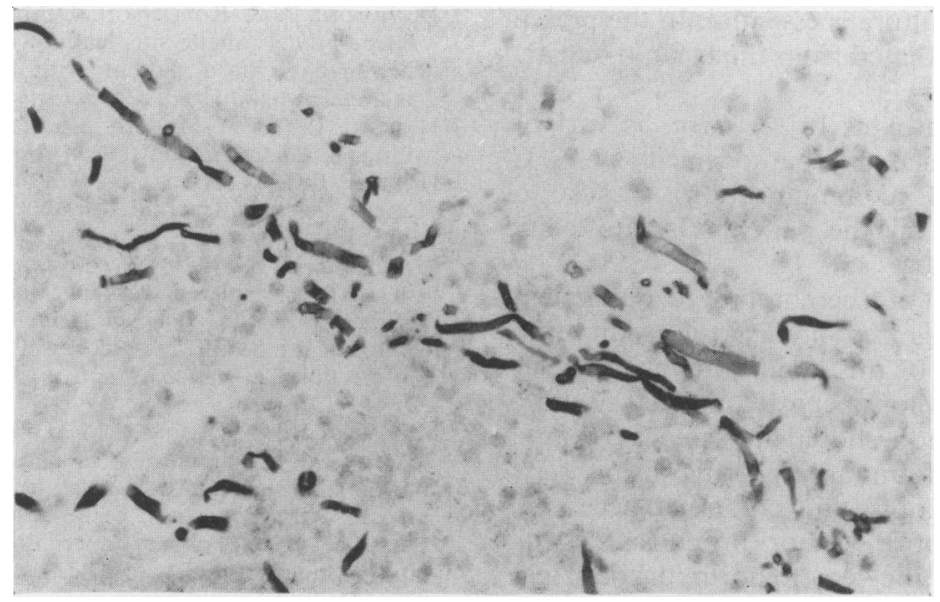

FIG. 2. Brain section (methenamine silver, $\times 160$ ). (Photographs by Miss M. Hudson, C.X.H.). Further illustrations of this case will be published later in a more detailed histopathology report.

dry and dusty conditions prevail for the greater part of the year, particularly in the North. Aspergillus flavus has been found in the dust and in vegetable matter, including timber, and it presumably exists in Saudia Arabia where climatic conditions are similar.

Inhalation of spores may produce pulmonary infection, particularly in damaged tissues, with haematogenous spread to the central nervous system, or infection of the nasal and paranasal sinuses may result in orbital invasion with extension into the brain. Apart from his cerebral abnormality this patient was a young man in general good health with no previous underlying or debilitating disease. There was no evidence of a primary focus of infection in the lungs and it was assumed that the solitary intracranial brain lesion was the result of entry and spread of the fungus via the upper respiratory passages during his occupation as a carpenter.

Laboratory diagnosis of the aspergilloma was made by the presence of hyphae in stained biopsy material, coupled with cultures which yielded profuse growth of $A$. flavus and confirmation by immunological methods. The presence of precipitating antibodies was determined by both double diffusion and counter-immunoelectrophoresis techniques using a wide range of antigens of various Aspergillus spp. The patient's own isolate gave the strongest reaction. Multiple precipitin lines are regarded as specific for aspergilloma and precipitin tests are a useful diagnostic procedure (Mahgoub, 1971).

The prognosis for cerebral aspergilloma is generally poor, although surgical removal and drainage may give temporary improvement, and sometimes cure, particularly when coupled with administration of antimycotic agents. Amphotericin B has been regarded generally as the drug of choice for effective therapy (Mukoyama, Gimpel and Posser, 1969), although in recent years the imidazoles have been tried, being less toxic and more acceptable to the patient. The patient's isolate of $A$. flavus appeared to be fairly resistant to amphotericin $B$ by in vitro tests and would indicate that a relatively high dose was required for intraventricular irrigation, even with additional intravenous administration (Atkinson and Bennett, 1978). Despite variations in determining the in vitro activity of the imidazoles (dependent on inoculum size, incubation time and medium used - Plempel, 1979; Haller, 1979) comparative tests by 2 laboratories indicated that econazole was more effective than miconazole against the A. flavus isolate (there being a 10-fold difference). Unfortunately the patient died before enconazole could be administered and its clinical value assessed.

There is little doubt that death was due to a massive intracranial haemorrhage following rupture of an arterial wall invaded by the hyphae of $A$. flavus. Detection of mycotic aneurysm in the brain is rare and only 5 cases associated with fungi have been reported previously - all confirmed at postmortem and not during life. Only once was the fungus isolated - A. fumigatus (Horten, Abbott and Porro, 1976). Aspergillus spp. were implicated by microscopy of the tissues (Davidson and Robertson, 1971; Visudhiphan et al., 1973; Mohandas et al., 1978), although the hyphae were more consistent with a Penicillium in the first case described by Morriss and Spock (1970). According to the morphological features of filamentous fungi in tissues, with lack of spore forms, other fungi such as Paecilomyces and Cephalosporium could also be 
implicated - hence culture is essential. In the present case, $A$. flavus was isolated from the brain tissue and confirmed by serology.

Production of aflatoxins by $A$. flavus is variable and not necessarily related to pathogenicity (P. K. C. Austwick, personal communication). Lack of aflatoxin production by the patient's isolate would seem to confirm this.

The insidious onset of a cerebral mycosis, particularly without a recognized predisposing condition, makes the diagnosis of this condition very difficult. Any patient who develops persistent orbital swelling, pain and loss of vision should be screened for the possible presence of an aspergilloma using serological tests, with biopsy and culture if necessary, especially in view of the poor prognosis of this disease. The use of the less toxic imidazoles, e.g. econazole, in management is suggested.

\section{Acknowledgments}

Many people have been involved in the diagnosis and management of this case. In particular, we would like to thank Dr F. Clifford Rose (Neurology), Mr R. D. Illingworth (Neurosurgery) and Prof. W. Blackwood (Neuropathology) of Charing Cross Hospital, Dr C. K. Campbell (Mycological Reference Lab., London School of Hygiene and Tropical Medicine), Dr R. Y. Cartwright (PHL, Guildford), Dr A. Onions (Commonwealth Mycological Institute, Kew), $\mathrm{Mr}$ P. K. C. Austwick (Brompton Hospital, Frimley) and members of their departments.

\section{References}

Atkinson, A.J. \& Bennetr, J.E. (1978) Amphotericin B pharmacokinetics in humans. Antimicrobial Agents and Chemotherapy, 13, 271.
DAvidson, P. \& Robertson, D.M. (1971) A true mycor (Aspergillus) aneurysm leading to fatal subarachno hemorrhage in a patient with hereditary hemorrhage telangiectasia. Journal of Neurosurgery, 35, 71.

Haller, I. (1979) Modern aspects of testing azole an fungals. Postgraduate Medical Journal, 55, 681.

Horten, B.C., AbBotT, G.F. \& Porro, R.S. (1976) Funge aneurysms of intracranial vessels. Archives of Neurolog 33, 577.

MAHGOUB, EL S. (1971) Mycological and serological studi of A. flavus isolated from paranasal aspergilloma in the Sudan. Journal of Tropical Medicine and Hygiene, 74, $16 \%$

Mahgoub, EL S. (1977) Mycoses of the Sudan. Transactions

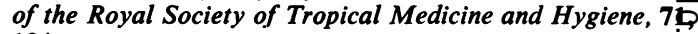
184.

Miloshev, B., Davidson, C.M., Gentles, J.C. \& Sandisov A.T. (1966) Aspergilloma of paranasal sinuses and orbip in Northern Sudanese. Lancet, i, 746.

Miloshev, B., Mahgoub, El S., Abdel Aal, O. \& Hassa疋 EL A.M. (1969) Primary aspergilloma of paranasal sinuses. in the Sudan: a review of 17 cases. British Journal of Surgery, 56, 132.

Mohandas, S., Ahuja, G.K., Sood, V.P. \& Virmani, Ф (1978) Aspergillosis of the central nervous system. Journal of the Neurological Sciences, 38, 299.

MORRISs, F.H. \& SPOCK, A. (1970) Intracranial aneurysm secondary to mycotic orbital and sinus infection. Americar Journal of Diseases of Children, 119, 357.

Mukoyama, M., Gimple, K. \& Posser, C.M. (1969) Aspef gillosis of the central nervous system. Report of a brai abscess due to $A$. fumigatus and review of the literature. Neurology. Minneapolis, 19, 967.

Plempel, M. (1979) Pharmacokinetics of imidazole 角t mycotics. Postgraduate Medical Journal, 55, 662.

Venugopal, P.V., Venugopal, T.V., ThiRUNeEklakan K., Subramanian, S. \& Shetty, B.M.V. (1977) Cerebra aspergillosis: report of two cases. Sabouradia, 15, 225.

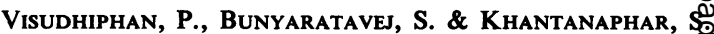
(1973) Cerebral aspergillosis: report of three cases. Journa of Neurosurgery, 38, 472. 